\section{A Feasibility Study for in vivo Dosimetry Procedure in Routine Clinical Practice}

\author{
Maria D. Falco, PhD $^{\top} \mathbb{D}$, Stefano Giancaterino, MSc', \\ Andrea De Nicola, MSc', Nico Adorante, MSc', \\ Ramon Gimenez De Lorenzo, MSc', Monica Di Tommaso, MD', \\ Annamaria Vinciguerra, MD', Marianna Trignani, MD', \\ Francesca Perrotti, MD', Albina Allajbej, MD', Andrea Fidanzio, PhD², \\ Francesca Greco, PhD $^{2}$, Mattia Grusio, $\mathbf{M S c}^{2}$, \\ Domenico Genovesi, MD', and Angelo Piermattei, PhD ${ }^{2}$
}

Technology in Cancer Research \&

Treatment

Volume 17: 1-8

(C) The Author(s) 2018

Reprints and permission:

sagepub.com/journalsPermissions.nav DOI: $10.1177 / 1533033818779201$ journals.sagepub.com/home/tct

(S)AGE

\begin{abstract}
Purpose: The aim of the in vivo dosimetry, during the fractionated radiation therapy, is the verification of the correct dose delivery to patient. Nowadays, in vivo dosimetry procedures for photon beams are based on the use of the electronic portal imaging device and dedicated software to elaborate electronic portal imaging device images. Methods: In total, 8474 in vivo dosimetry tests were carried out for 386 patients treated with 3-dimensional conformal radiotherapy, intensity-modulated radiotherapy, and volumetric modulated arc therapy techniques, using the SOFTDISO. SOFTDISO is a dedicated software that uses electronic portal imaging device images in order to $(I)$ calculate the $R$ index, that is, the ratio between daily reconstructed dose and the planned one at isocenter and (2) perform a $\gamma$-like analysis between the signals, S, of a reference electronic portal imaging device image and that obtained in a daily fraction. It supplies 2 indexes, the percentage $\gamma \%$ of points with $\gamma<\mathrm{I}$ and the mean $\gamma$ value, $\gamma_{\text {mean }}$. In $\gamma$-like analysis, the pass criteria for the signals agreement $\Delta \mathrm{S} \%$ and distance to agreement $\Delta \mathrm{d}$ have been selected based on the clinical experience and technology used. The adopted tolerance levels for the 3 indexes were fixed in $0.95 \leq$ $\mathrm{R} \leq \mathrm{I} .05, \gamma \% \geq 90 \%$, and $\gamma_{\text {mean }} \leq 0.5$. Results: The results of $\mathrm{R}$ ratio, $\gamma$-like, and a visual inspection of these data reported on a monitor screen permitted to individuate 2 classes of errors (I) class I that included errors due to inadequate standard quality controls and (2) class 2, due to patient morphological changes. Depending on the technique and anatomical site, a maximum of $18 \%$ of tests had at least I index out of tolerance; once removed the causes of class-I errors, almost all patients (except patients with 4 lung and 2 breast cancer treated with 3-dimensional conformal radiotherapy) presented mean indexes values ( $\bar{R}, \bar{\gamma} \%$, and $\left.\bar{\gamma}_{\text {mean }}\right)$ within tolerance at the end of treatment course. Class- 2 errors were found in some patients. Conclusions: The in vivo dosimetry procedure with SOFTDISO resulted easily implementable, able to individuate errors with a limited workload.
\end{abstract}

\title{
Keywords
}

3-DCRT, IMRT, VMAT, in vivo dosimetry, EPID imaging, $\gamma$-like analysis

\section{Abbreviations}

aSi-EPID, amorphous-silicon electronic portal imaging device; au, arbitrary unit; CBCT, cone beam computed tomography; CT, computed tomography; 2D, 2 dimensional; 3-DCRT, 3-dimensional conformal radiotherapy; DICOM, Digital Imaging and COmmunications in Medicine; DRR, digitally reconstructed radiograph; EPID, electronic portal imaging device; IMRT,

\footnotetext{
'Department of Radiation Oncology “G. D’Annunzio”, University of Chieti, SS. Annunziata Hospital, Chieti, Italy

${ }^{2}$ Unità Operativa di Fisica Sanitaria; Fondazione Policlinico Universitario A. Gemelli, Università Cattolica del Sacro Cuore, Roma, Italy
}

Corresponding Author:

Maria D. Falco, PhD, Department of Radiation Oncology “G. D’Annunzio”, University of Chieti, SS. Annunziata Hospital, Chieti, Italy. Email: daniela.falco@asl2abruzzo.it 
intensity-modulated radiotherapy; IVD, in vivo dosimetry; LINAC, linear accelerator; MLC, multileaf collimator; MU, monitor unit; QC, quality control; RT, radiotherapy; TPR, tissue phantom ratio; TPS, treatment planning system; VMAT, volumetric modulated arc therapy.

Received: July 28, 2017; Revised: December 21, 2017; Accepted: January 26, 2018.

\section{Introduction}

During radiotherapy treatment, dosimetric differences between planned and delivered doses can cause treatment failure. Generally an adequate quality control (QC) procedure, adopted in the radiotherapy centres, is able to alleviate dosimetric errors. However, during the fractionated dose delivery, the planned dose can be different from the prescribed one ${ }^{1}$ generally due to inadequate $\mathrm{QC}$. In extreme cases, an inadequate $\mathrm{QC}$ program has led to major incidents with severe complications for patients, as largely reported in the literature. ${ }^{2-6}$ The in vivo dosimetry (IVD) is strongly recommended by various organizations $^{7-10}$ so that in some countries, IVD tests are required as a routine check to detect the causes of dosimetric errors. ${ }^{11,12}$ Several researchers ${ }^{13-35}$ have implemented IVD systems to reconstruct the delivered dose to the patients using the amorphous silicon electronic portal imaging device (aSi-EPID) associated with the linear accelerator (LINAC). The results showed that aSi-EPIDs can be used to reconstruct the dose in single points, 2 dimensional (2D), and entire 3-dimensional (3D) dose distribution.

One of the major issues for the application of an IVD procedure in clinical practice is high workload. A practical IVD procedure should (1) assure an acceptable workload, (2) examine an adequate number of patients tested every day, (3) provide a new IVD acquisition in the subsequent day to verify the efficacy of the adopted corrections.

The aim of the study was to test the feasibility of the IVD procedure, carried out with a practical software, to intercept and eventually correct the errors during the delivery in order to improve treatment reproducibility.

\section{Materials and Methods}

\section{Linear Accelerator and Treatment Planning System}

An Elekta Synergy Agility LINAC (Crawley, United Kingdom) with a 160 Multileaf Collimators (MLC) is operative at the University of Chieti, SS. Annunziata Hospital, to carry out 3-dimensional conformal radiotherapy (3-DCRT), intensity-modulated radiotherapy (IMRT), and volumetric modulated arc therapy (VMAT) radiotherapy (RT) treatments using $6 \mathrm{MV}$ and $10 \mathrm{MV}$ beams. The LINAC is equipped with a cone beam computed tomography (CBCT) scanner (version 5.18.0) and an Elekta IviewGT EPID, based on aSi panels XRD 1640 AL5 (PerkinElmer Optoelectronics, Fremont, California) operating as a 2-D photodiode array at the fixed Source-EPID of $159 \mathrm{~cm}$. The sensitive layer of EPID consists of $1024 \times 1024$ pixels with a pitch of $400 \mu \mathrm{m}$, resulting in an active area of $409.6 \times 409.6 \mathrm{~mm}^{2}$. The treatments were performed using a robotic couch (iBEAMevo, Elekta, Crawley, United Kingdom) for the automatic patient repositioning considering only translational displacements. All radiotherapy plans were carried out using Oncentra External Beam treatment planning system (TPS) v.4.3 (Nucletron BV, Veenendaal, the Netherlands). Dose calculation was performed using the collapsed cone convolution algorithm with inhomogeneity correction, using a cubic grid resolution of $2 \mathrm{~mm}$.

\section{Enrolled Patients}

Table 1 reports the baseline characteristics of 386 patients treated with 3-DCRT, IMRT, and VMAT techniques for various anatomical treatment sites. For the 3 techniques, optimized patient setups were obtained by the CBCT images to correct eventual out-of-tolerance patient position. This setup was carried at first 3 treatment fractions and then once a week; exception for breast treatments with 3-DCRT, for which optimized patient setups were obtained by the comparison between 2 orthogonal megavoltage images (medial and lateral) and the digitally reconstructed radiographs (DRRs) images, at the first 3 treatment fractions and then once a week. In all other treatment sessions, daily patient setup was obtained by a current setup based on the laser beam alignment on the patient tattoos. For head neck $(\mathrm{H} \& \mathrm{~N})$ and brain treatments, thermoplastic masks were used to immobilize the patient during treatment.

Table 2 reports the maximum accepted variations in $x, y$, and $z$ coordinates for all the techniques, indicated by a unique symbol $\Delta \mathrm{r}$. They are based on the optimized patient setup verification system (CBCT or EPID) and the complexity of the technique delivery and immobilization systems. In particular, the data reported in Table 2 take into account situations where the patient current setup reproducibility was difficult to reach.

This study was carried out after informed consent had been given by the patients.

\section{SOFTDISO Commissioning and Application}

SOFTDISO is a dedicated software for IVD first developed by a National Italian Project ${ }^{27}$ and then delivered by Best Medical Italy, Chianciano, Italy. It can be applied at 3-DCRT, IMRT, and VMAT techniques for different anatomical treatment sites. Its commissioning for LINAC equipped with aSi-EPID requires the following measurements for each beam quality: 
Table 1. Baseline Characteristics of 386 Patients Examined in Terms of Number of Anatomical Sites $(\mathrm{n} \%)$ and Techniques Number N.

\begin{tabular}{lc}
\hline Anatomical sites & $\mathrm{n}(\%)$ \\
\hline Lung & 10 \\
Pelvis & 27 \\
Breast & 10 \\
Abdomen & 4 \\
Head neck & 12 \\
Prostate & 37 \\
\hline Techniques & $\mathrm{N}$ \\
\hline 3-DCRT & 134 \\
IMRT & 78 \\
VMAT & 174 \\
\hline
\end{tabular}

Abbreviations: 3-DCRT, 3-dimensional conformal radiotherapy; IMRT, intensity-modulated radiotherapy; VMAT, in vivo dosimetry.

Table 2. Maximum Accepted Variations $(\Delta r)$ for the Set-Up Reproducibility in All Techniques for Each Anatomical Site. ${ }^{a}$

\begin{tabular}{|c|c|c|c|}
\hline \multirow{2}{*}{$\begin{array}{l}\text { Technique } \\
\text { Accepted variations }\end{array}$} & 3-DCRT & IMRT & VMAT \\
\hline & $\Delta \mathrm{r}(\mathrm{mm})$ & $\Delta \mathrm{r}(\mathrm{mm})$ & $\Delta \mathrm{r}(\mathrm{mm})$ \\
\hline Lung & 5 & - & - \\
\hline Pelvis & 5 & 5 & - \\
\hline Abdomen & 5 & - & - \\
\hline Head and neck & - & 3 & 3 \\
\hline Breast & 5 & - & - \\
\hline Prostate & - & - & 3 \\
\hline
\end{tabular}

Abbreviations: 3-DCRT, 3-dimensional conformal radiotherapy; IMRT, intensity-modulated radiotherapy; VMAT, in vivo dosimetry.

${ }^{\mathrm{a}}$ The dashes stand for the techniques not used for each tumor site.

- the LINAC output factor $\mathrm{F}(\mathrm{Gy} / \mathrm{MU})$ at the reference depth in water ${ }^{36}$;

- the index beam quality $\mathrm{TPR}_{10}^{2036}$;

- the EPID calibration factor $\mathrm{k}_{\mathrm{s}}^{27}$;

- the EPID linearity factor $\mathrm{k}_{\mathrm{lin}}{ }^{27}$.

The $\mathrm{F}$ and $\mathrm{TPR}_{10}^{20}$ parameters are obtained during the LINAC commissioning and periodically verified in the Centre. $\mathrm{k}_{\mathrm{s}}$ and $\mathrm{k}_{\mathrm{lin}}$ require specific measurements (in terms of EPID arbitrary unit per LINAC-Monitor Unit, ie, au/MU) and generally are carried out in about 30 minutes for each beam quality. The factor $\mathrm{k}_{\mathrm{s}}$ is defined as the ratio between $(1 \mathrm{au} / 100 \mathrm{MU})$ and the EPID signal $\mathrm{S}(\mathrm{au} / 100 \mathrm{MU})$, measured on the beam central axis with the EPID at $159 \mathrm{~cm}$ from the source. $\mathrm{k}_{\text {lin }}$ takes into account the dependence of $\mathrm{k}_{\mathrm{s}}$ as a function of the number of MUs used. We tested $\mathrm{k}_{\mathrm{s}}$ and $\mathrm{k}_{\text {lin }}$ dependence on the dose rate at baseline and we found it negligible (within $\pm 0.5 \%$ ). Since EPID signals can change in time, then $\mathrm{k}_{\mathrm{s}}$ and $\mathrm{k}_{\text {lin }}$ were tested once a month and once a year, respectively. ${ }^{27,37}$

SOFTDISO is interfaced with Oncentra TPS via Digital Imaging and COmmunications in Medicine. It uses computed tomography (CT) scans and the RTPlan to generate the patient radiological thicknesses, the beam geometry, and the isocenter dose by TPS ( $D_{\text {iso, TPS }}$ ), for each beam. The software is also directly interfaced with the IVIEWGT portal imaging system, and data are recorded in DICOM-RT files and stored in SOFTDISO.

The integral EPID image, referred to each single static or dynamic beam of the treatment fraction, is elaborated to determine:

- the $\mathrm{R}$ ratio between the reconstructed isocenter dose $\left(D_{\text {iso, EPID }}\right)$ by EPID image and that planned by TPS,

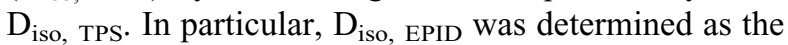
average reconstructed dose values obtained from the points in $\Delta \mathrm{r}$ side square area (around the beam central axis). The dose reconstruction algorithms for the 3 techniques used are reported in the literature, ${ }^{27-35}$ where the global accuracy for the $\mathrm{R}$ ratio is determined considering both the $\mathrm{D}_{\text {iso, EPID }}$ accuracy (in inhomogeneous tissues estimated in $4 \%)^{13,28-34}$ and the $\mathrm{D}_{\text {iso, TPS }}$ accuracy (generally estimated well within $3 \%$ ). The statistical propagation of the uncertainties supplies for $\mathrm{R}$ ratio index the tolerance level $0.95 \leq \mathrm{R} \leq 1.05$;

- $\gamma$-Like analysis which is the comparison between the EPID signal, S, of the reference image (after the optimized patient setup) and that of the EPID image measured with current patient setup. For the $\gamma$-like analysis, the methodological approach is the same as the $\gamma$-analysis. ${ }^{38}$ However, $\gamma$-like analysis is based on signal discrepancies, while $\gamma$-analysis is carried out on the dosimetric discrepancies. The pass criteria for $\gamma$-like analysis were (1) distance to agreement $\Delta \mathrm{d}$ equal to $\Delta \mathrm{r}$ (Table 2), (2) signal agreement $\Delta \mathrm{S} \%$ selected from $3 \%$ to $5 \%$ depending on the presence of inhomogeneous tissues, technique used, and mobility of the irradiated organs. ${ }^{35}$ For example, treatments in the presence of homogeneous tissues and thermoplastic mask in VMAT treatments, $\Delta \mathrm{d}=3 \mathrm{~mm}$ and $\Delta \mathrm{S}=3 \%$ and $\Delta \mathrm{d}=5 \mathrm{~mm}$ and $\Delta \mathrm{S}=5 \%$, were used in the presence of inhomogeneous tissues and difficult reproducibility due to patient positioning. Tolerance levels were fixed for (1) the percentage $\gamma \% \geq 90 \%$ (ie, the percentage of points with $\gamma<1$ must be greater or equal to $90 \%$ ) and (2) the mean of the $\gamma$ values, $\gamma_{\text {mean }} \leq 0.5$. High $\gamma_{\text {mean }}$ index values indicated probably organ shifts that were more pronounced especially in the presence of different media, such as air/muscle (gas pockets in rectum), lung/muscle (presence of lung tumor regression or atelectasys), and bone/muscle (vertebras shift).

All the above reported threshold values were chosen in agreement to previous literature data. ${ }^{14,28-35}$

The cause of discrepancies was summarized in 2 classes:

- Class 1, that is, errors due to inadequate standard QC, as patient setup, presence of attenuators on the beam not included in TPS calculation (as some parts of the couch 
Table 3. Results of IVD Tests for 3DCRT Techniques Applied to 4 Treatment Sites. ${ }^{\text {a }}$

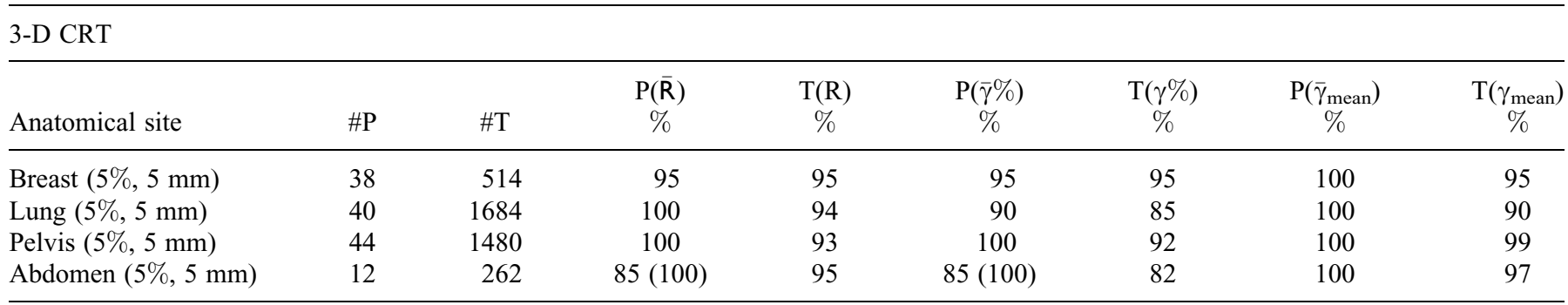

Abbreviations: 3-DCRT, 3-dimensional conformal radiotherapy.

${ }^{\mathrm{a}} \mathrm{AP}$ and \#T are the number of patients and tests, respectively. $\mathrm{P}(\overline{\mathrm{R}}), \mathrm{P}(\bar{\gamma} \%)$, and $\mathrm{P}\left(\bar{\gamma}_{\text {mean }}\right)$ correspond to the percentages of patients $(\mathrm{P})$ within tolerance $\overline{\mathrm{R}}, \bar{\gamma} \%$, and $\bar{\gamma}_{\text {mean }}$ and $\mathrm{T}(\mathrm{R}), \mathrm{T}(\gamma \%)$, and $\mathrm{T}\left(\gamma_{\text {mean }}\right)$ are the percentages of tests $(\mathrm{T})$ within tolerance R, $\gamma \%$, \% and $\gamma_{\text {mean }}$, respectively. Of all, 355 of 3940 tests $(9 \%)$ resulted in OTL. For the abdomen treatments, 2 percentage values are reported; the first 1 corresponds to the percentage considering all the errors (class 1 and class 2 ); the second was obtained omitting class-2 errors.

Table 4. Results of IVD Tests for IMRT Techniques Applied to 2 Anatomical Sites. ${ }^{\text {a }}$

\begin{tabular}{lccccccc}
\hline IMRT & & & & & \\
\hline Anatomical site & $\# \mathrm{P}$ & $\# \mathrm{~T}$ & $\begin{array}{c}\mathrm{P}(\overline{\mathrm{R}}) \\
\%\end{array}$ & $\begin{array}{c}\mathrm{T}(\mathrm{R}) \\
\%\end{array}$ & $\begin{array}{c}\mathrm{P}(\bar{\gamma} \%) \\
\%\end{array}$ & $\begin{array}{c}\mathrm{T}(\gamma \%) \\
\%\end{array}$ & $\begin{array}{c}\mathrm{P}\left(\bar{\gamma}_{\text {mean }}\right) \\
\%\end{array}$ \\
\hline Pelvis $(5 \%, 5 \mathrm{~mm})$ & 60 & 1624 & 100 & 95 & 100 & 93 & 100 \\
H\&N $(3 \%, 3 \mathrm{~mm})$ & 18 & 1100 & 100 & 98 & 100 & 93 & 99 \\
\hline
\end{tabular}

Abbreviations: H\&N, head neck; IMRT, intensity-modulated radiotherapy.

${ }^{\mathrm{a}} \# \mathrm{P}$ and \#T are the number of patients and tests, respectively. $\mathrm{P}(\overline{\mathrm{R}}), \mathrm{P}(\bar{\gamma} \%)$, and $\mathrm{P}\left(\bar{\gamma}_{\text {mean }}\right)$ correspond to the percentages of patients $(\mathrm{P})$ within tolerance $\overline{\mathrm{R}}, \bar{\gamma}$, and $\bar{\gamma}_{\text {mean }}$ and $\mathrm{T}(\mathrm{R}), \mathrm{T}(\gamma \%)$, and $\mathrm{T}\left(\gamma_{\text {mean }}\right)$ are the percentages of tests (T) within tolerance R, $\gamma \%$, and $\gamma_{\text {mean }}$, respectively. Of all, 191 (7\%) of 2724 tests resulted in OTL.

that could be intercepted by the beams) and incorrect data transfer;

- Class 2, that is, errors due to patient morphological changes such as tumor shrinkage, patient weight loss, atelectasis, and gas pockets that required specific controls during the treatment.

For each patient, IVD test supplied the results of $\mathrm{R}$ ratio, $\gamma \%$, and $\gamma_{\text {mean }}$ for every static or dynamic beam used in the treatment fraction. These tests were performed in the following 2 days after optimized patient setup in the first week of treatment, and then once a week after the current patient setup. The results of 3 indexes values $\left(\mathrm{R}, \gamma \%\right.$, and $\left.\gamma_{\text {mean }}\right)$ were automatically stored in the SOFTDISO database. If one index resulted out of tolerance level (OTL; ie, $\mathrm{R}<0.95$ or $\mathrm{R}>1.05, \gamma \%<90$, and $\left.\gamma_{\text {mean }}>0.5\right)$, it was indicated in red. Subsequently, an accurate analysis of OTL results on the monitor screen, as will be discussed talking about Figure 2 of the "Discussion" section, allowed to individuate 2 class of errors. These errors were analyzed by a medical physicist and successively by the radiation oncologist in order to find the adequate corrections to the discrepancies found; a new IVD test was performed to verify the efficacy of the adopted corrections the day after. For each treatment fraction, SOFTDISO supplies the mean values of R, $\gamma \%$, and $\gamma_{\text {mean }}$ for every patient. These mean values $\overline{\mathrm{R}}, \bar{\gamma} \%$, and $\bar{\gamma}_{\text {mean }}$ at the end of treatment course are provided for all tests too, and the aim of the corrective actions was to obtain all in-tolerance mean index values.
About the workload, in our institute, 10 patients per day are subjected to IVD tests. Considering that EPID images processing and loading requires about 5 minutes, and the time necessary for visual inspection of OTL tests is of about 4 minutes for patient, and a maximum of 4 patients with OTL tests, the daily workload was observed to be about 20 minutes per day. An extra time could be necessary when the cause of the error is not immediately recognized.

\section{Results}

Of all, 8474 IVD tests were carried out for 386 patients. The IVD results obtained for 3-DCRT, IMRT, and VMAT techniques are summarized in Tables 3, 4, and 5, respectively. For each treatment anatomical site in the first column, the pass criteria $\Delta \mathrm{S} \%$ and $\Delta \mathrm{d}$ used are reported; in the second and third column, the numbers of patients, \#P, and the IVD tests, \#T, are reported; the other columns report for each index the percentages of patients (P) within tolerance $\bar{R}, \bar{\gamma} \%$, and $\bar{\gamma}_{\text {mean }}\left(\mathrm{P}(\bar{R}), \mathrm{P}(\bar{\gamma} \%)\right.$, and $\left.\mathrm{P}\left(\bar{\gamma}_{\text {mean }}\right)\right)$ and the percentages of tests (T) within tolerance R, $\gamma \%$, and $\gamma_{\text {mean }}(\mathrm{T}(\mathrm{R}), \mathrm{T}(\gamma \%)$, and $\mathrm{T}\left(\gamma_{\text {mean }}\right)$ ), respectively. All percentages are approximated to integer numbers.

In total, 444 of 3940 tests for the 3-DCRT techniques (about $11 \%$ ) were in OTL. Two patients with pancreatic cancer (abdomen) and 2 patients with breast cancer presented $\bar{R}$ and $\bar{\gamma} \%$ OTL values due to occasional intestinal gas pockets and incorrect breast setups, respectively. In addition, for abdomen, 
Table 5. Results of IVD Tests for VMAT Techniques Applied to 2 Anatomical Sites. ${ }^{\text {a }}$

\begin{tabular}{|c|c|c|c|c|c|c|c|c|}
\hline \multicolumn{9}{|l|}{ VMAT } \\
\hline Prostate $(3 \%, 3 \mathrm{~mm})$ & 142 & 1378 & 100 & 96 & $96(100)$ & 90 & 100 & 96 \\
\hline $\mathrm{H} \& \mathrm{~N}(3 \%, 3 \mathrm{~mm})$ & 32 & 432 & 100 & 97 & 100 & 94 & 100 & 98 \\
\hline
\end{tabular}

Abbreviations: H\&N, head neck; VMAT, volumetric modulated arc therapy.

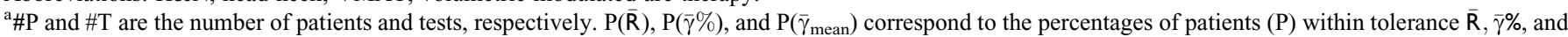

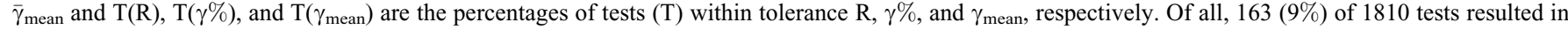

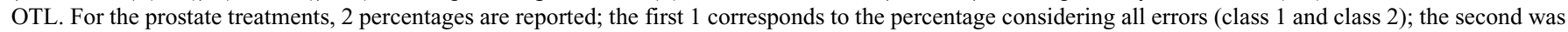
obtained omitting class- 2 errors.

2 percentage values for $\mathrm{P}(\bar{R})$ and $\mathrm{P}(\bar{\gamma} \%)$ are reported. The first one corresponds to the percentage considering both class- 1 and class- 2 errors; the second value (in parenthesis) is referred to OTL tests due to class- 1 errors, only. This means that the dedicated personnel (a technician and a medical physicist) identified tests containing the presences of abdomen gas pockets. The latter were classified as class- 2 errors and were omitted from statistic of the standard QCs. As a consequence, $\mathrm{P}(\bar{R})$ and $\mathrm{P}(\bar{\gamma} \%)$ reached $100 \%$ for almost all treatment. In vivo dosimetry tests of breast treatments presented $\mathrm{R}$ and $\gamma \%$ values out of tolerance (due to patient setup errors), and the subsequent intolerance number of tests was insufficient to obtain the mean index values within tolerance.

Only 2 lung treatments reported some OTL tests due to the presence of an attenuator (ie, the hooks of the couch extension intercepted some beams). Removing the couch extension, the tests presented values within the tolerances. Four patients with lung cancer showed OTL $\bar{\gamma} \%$ values. From $\gamma$-like analysis, shift in patient positioning was observed, and the discrepancies were due to incorrect patient setup. Although adequate corrections were adopted, that is, increasing the number of CBCTs and using a different immobilization system, $\bar{\gamma} \%$ remained out of tolerance due to the limited number of tests following the corrections. Moreover, small tumor shrinkages were randomly observed especially in the last radiotherapy fractions, thus they were not taken into account. In summary, for 3-DCRT technique, $2(5 \%)$ patients with breast cancer and $4(10 \%)$ patients with lung cancer presented at least 1 index in OTL due to class-1 errors.

Table 4 reports the results of 2724 tests for pelvis and H\&N tumors treated with IMRT technique with 191 (7\%) OTL tests . All patients showed $\mathrm{P}(\overline{\mathrm{R}}), \mathrm{P}(\bar{\gamma} \%)$, and $\mathrm{P}\left(\bar{\gamma}_{\text {mean }}\right)$ within the tolerance levels. The OTL tests were due to pelvis setup errors for 2 overweight patients. Occasional gas pockets were observed by the gamma analysis but they did not influence the final results of the 3 mean indexes.

Table 5 reports the results of 1810 tests for prostate and H\&N tumors treated with VMAT technique with $163(9 \%)$ OTL tests. If class- 2 errors were excluded, all patients showed $\mathrm{P}(\overline{\mathrm{R}}), \mathrm{P}(\bar{\gamma} \%)$, and $\mathrm{P}\left(\bar{\gamma}_{\text {mean }}\right)$ within the tolerance levels. Four patients with prostate cancer presented random OTL tests due to anatomical variations. In particular, 1 patient, due to the

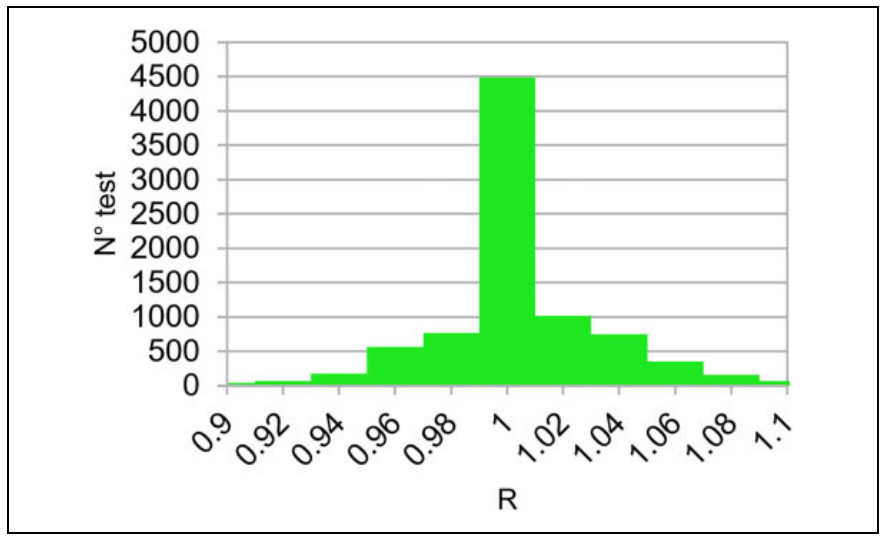

Figure 1. Distribution of R ratios obtained for the 8474 tests carried out for 386 patients treated with 3-DCRT, IMRT, and VMAT techniques. 3-DCRT indicates 3-dimensional conformal radiotherapy; IMRT, intensity-modulated radiotherapy; VMAT, in vivo dosimetry.

disease, presented a full rectum in the CT scans used for the planning. At mid-therapy, due to increased intestinal motion induced by radiotherapy, the rectum tended to empty and $\gamma$-like analysis showed OTL values due to the presence of air filling (ie, $R=1.05, \gamma \%=85 \%$, and $\gamma_{\text {mean }}=0.6$ were found in 1 test). The presence of air filling was confirmed by CBCT scans, too. A new CT scan was required for dose recalculation. As small dose variations (for both target coverage and rectum) were found, an adaptive plan was not taken into account.

One patient with H\&N cancer initially received RT delivery for PTV, and subsequently a boost dose of radiation was delivered using the same thermoplastic mask. At the first fraction of boost delivery, OTL test was observed due to weight loss causing an in-plane shift of patient setup. In this case, a new treatment $\mathrm{CT}$ simulation was acquired for the new plane using a new thermoplastic mask. The next IVD test showed in-tolerance index values.

Figure 1 shows histogram values of $\mathrm{R}$ ratios for all the 8474 tests corresponding to 386 patients studied in this work. The overall mean $\mathrm{R}$ ratio was equal to 1.004 (0.03), (1SD), and the dispersion of $\mathrm{R}$ ratios is quite symmetrical around 1 (Fisher skewness coefficient $=0.2, P<.0008) .{ }^{39}$ The distributions of the 3 indexes $\left(\mathrm{R}, \gamma \%\right.$, and $\left.\gamma_{\text {mean }}\right)$ resulted in good agreement with the results obtained by other researchers who 


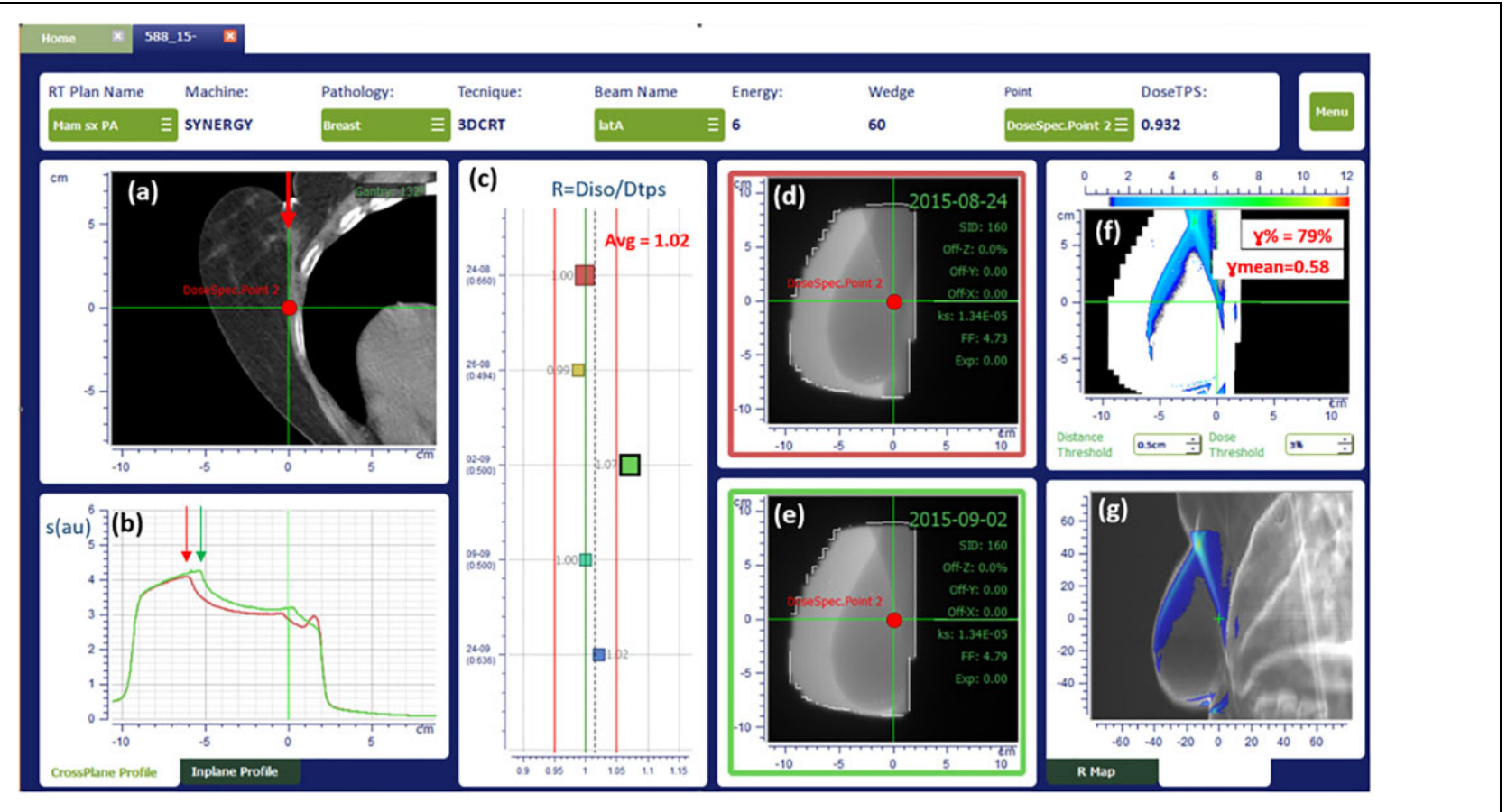

Figure 2. In vivo dosimetry results for a breast treatment with 3-DCRT, as displayed by SOFTDISO. 3-DCRT indicates 3-dimensional conformal radiotherapy.

developed similar software using the same tolerance levels. $^{16-18,29-34}$

\section{Discussion}

We reported the results of 8474 IVD tests obtained with SOFTDISO for 3-DCRT, IMRT, and VMAT techniques. Tests presenting class- 1 errors were intercepted, and a new IVD test was performed in the subsequent treatment fraction to verify the adopted corrections. When the right corrections were applied, all the 3 indexes $\left(\mathrm{R}, \gamma \%\right.$, and $\left.\gamma_{\text {mean }}\right)$ went back within tolerance. We have estimated that in absence of the corrections, the percentage of OTL tests ( $\mathrm{T} \%$ ) could reach about 37\%. In vivo dosimetry procedure was able to identify the errors which were corrected in the subsequent day. Therefore, the percentage of OTL test range values were $1 \%$ to $18 \%$ for 3 -DCRT, $1 \%$ to $7 \%$ for IMRT, and $2 \%$ to $10 \%$ for VMAT.

In particular, 2 patients with breast cancer treatments and 4 patients with lung cancer treated with 3-DCRT presented at least 1 mean index $\left(\overline{\mathrm{R}}, \bar{\gamma} \%\right.$, and $\left.\bar{\gamma}_{\text {mean }}\right)$ in OTL due to insufficient number of tests following the corrections. These results are not able to provide information about the discrepancy between overall planned and delivered dose, but they can only figure out the presence of errors that can be rapidly removed. For breast treatments, the worst results were observed due to excessive mobility caused by large breast volumes or by random interception of the beam by the couch-edges. In order to remove the latter source of error, new directions of the beams were adopted, and a new treatment plan was elaborated. Better results were obtained by the collaboration of the medical team including medical physicists, radiation oncologist, and radiological technicians.

An example of SOFTDISO interface that shows OTL results for a tangential breast treatment using a wedged beam of $60^{\circ}$ is presented in Figure 2 where data of the IVD test are showed. In particular, the software reports in (1) the patient CT scan (containing the isocenter point) with incident beam emerging from the top (red arrow), (2) the comparison between EPID signal profile (in red) of the reference EPID image as reported in section (d), and the profile (in green) obtained in the third IVD test as showed in section (e) along the cross plane axis, (3) the $\mathrm{R}$ ratios obtained in 5 different treatment days, (4) and (5) report the reference (contoured in a red square) and current EPID images (contoured in a green square), respectively, (6) a map of points with $\gamma \geq 1$ and (7) the map on the different projections of patient DRR image. Anatomical region presenting the disagreement is clearly identified in the last section $(\mathrm{g})$. The 2 arrows indicate a patient setup error due to breast displacement of about $1 \mathrm{~cm}$ causing $R=1.07, \gamma \%=79 \%$, and $\gamma_{\text {mean }}=0.58$ as reported in the sections (c) and (f), corresponding to index values of the third treatment fraction for the beam under investigation. Once patient setup was corrected, all the mean indexes returned within tolerance.

To identify classe- 1 or class- 2 errors, the dedicated personnel analyzed the results showed on the SOFTDISO interface that are similar to those reported in Figure 2, with particular attention to (1) EPID signal profiles along in plane or cross plane axes (Figure 2B) to verify the presence of patient setup errors or the presence of beam attenuators and (2) the position of the map of points with $\gamma \geq 1$ on the DRR images (Figure 2G) 
to verify the presence of morphological changes; the later can be random (gas pockets) or the progressive changes in the tissues (H\&N tissue variations or lung tumor shrinkages).

\section{Conclusions}

The results reported in this article showed that the SOFTDISO practical IVD procedure was able to intercept errors during treatment fractions and to verify the appropriateness of the eventual corrections in the subsequent radiotherapy session. It permits to examine an adequate number of patients tested every day requiring a limited daily workload. Therefore, it can be easily introduced in the clinical routine as a part of the quality assurance program. For further improvement in IVD procedure, the authors intend to (1) use personalized $\Delta \mathrm{d}$ values for each patient. For patients presenting a more reproducible setup, pass criteria should be more restrictive than those reported in Table 2; even though this can increase the workload, it can reveal other kinds of errors, such as class2 errors; (2) daily use of the CBCT to calculate R ratio. Currently, some IVD procedures perform dose reconstruction using planning CT scan. This means that in case of IVD warnings, for example, due to incorrect patient setup or anatomical changes, the reconstructed photon fluence on planning CT may differ from that used for the dose delivery. Therefore, IVD tests based on single points, 2-D, or 3-D dose recalculation can present some inaccuracies. It is a general opinion ${ }^{40,41}$ that the use of CBCT scans for the reconstruction of the dose delivery using EPID images could improve the accuracy of the reconstructed dose, but the CBCT image calibration methods need further optimization.

\section{Declaration of Conflicting Interests}

The author(s) declared no potential conflicts of interest with respect to the research, authorship, and/or publication of this article.

\section{Funding}

The author(s) received no financial support for the research, authorship, and/or publication of this article.

\section{ORCID iD}

Maria D. Falco, PhD (D http://orcid.org/0000-0002-4454-5403

\section{References}

1. Mijnheer B, Beddar S, Izewska J, Reft C. In vivo dosimetry in external beam radiotherapy. Med Phys. 2013;40(7):070903.

2. Mayles W. The Glasgow incident-a physicist's reflections. Clin Oncol. 2007;19(1):4-7.

3. Williams MV. Radiotherapy near misses, incidents and errors: radiotherapy incident in Glasgow. Clin Oncol. 2007;19(1):1-3.

4. Bogdanovich W. As technology surges, radiation safety lag. Radiation offers new cures and ways to do harm. Radiation therapy's harmful Side. New York Times. 23 January 2010.

5. Bogdanovich W. As technology surges, radiation safety lag. As technology surges, radiation safeguards lag. New York Times. 26 January 2010.
6. Bogdanovich W. As technology surges, radiation safety lag. A pinpoint beam strays invisibly, harming instead of healing. New York Times. 29 December 2010.

7. Ortiz López P, Cosset JM, Dunscombe P, et al. International Commission on Radiological Protection (ICRP). Preventing accidental exposures from new external beam radiation therapy technologies. Ann ICRP. 2009;39(4):1-86.

8. American Association of Physicists in Medicine (AAPM). Diode in vivo dosimetry for patients receiving external beam radiotherapy [AAPM Report No. 87]. Madison, WI: Medical Physics Publishing; 2005.

9. International Atomic Energy Agency (IAEA). Development of procedures for in vivo dosimetry in radiotherapy. IAEA Human Health Report. 2013;8.

10. The Swedish Radiation Protection Institute (SSI). Regulations on Radiation Therapy. Report SSI FS 2000:4 The Swedish Radiation Protection Institute: Stockholm, Sweden, 2000.

11. Derreumaux S, Etard C, Huet C, et al. Lessons from recent accidents in radiation therapy in France. Radiat Prot Dosim. 2008; 131(1):130-135.

12. The Royal College of Radiologists, Society and College of Radiographers, Institute of Physics and Engineering in Medicine, British Institute of Radiology. Implementing In Vivo Dosimetry. Royal College of Radiologists: London, 2008.

13. Piermattei A, Fidanzio A, Azario L, et al. Application of a practical method for the isocenter point in vivo dosimetry by a transit signal. Phys Med Biol. 2007;52(16):5101-5117.

14. Bedford JL, Hanson IM, Hansen VN. Portal dosimetry for VMAT using integrated images obtained during treatment. Med Phys. 2014;41(2):021725.

15. Francois $\mathrm{P}$, Boissard $\mathrm{P}$, Berger L, Mazal A. In vivo dose verification from back projection of a transit dose measurement on the central axis of photon beams. Phys Med. 2011;27(1):1-10.

16. Camilleri J, Mazurier J, Franck D, Dudouet P, Latorzeff I, Franceries X. Clinical results of an EPID-based in-vivo dosimetry method for pelvic cancers treated by intensity-modulated radiation therapy. Phys Med. 2014;30(6):690-695.

17. van Elmpt W, McDermott L, Nijsten S, Wendling M, Lambin P, Mijnheer B. A literature review of electronic portal imaging for radiotherapy dosimetry. Radiother Oncol. 2008;88(3):289-309.

18. Nijsten S, van Elmpt W, Jacobs M, et al. A global calibration model for a-Si EPIDs used for transit dosimetry. Med Phys. 2007;34(10):3872-3884.

19. Mans A, Wendling M, McDermott LN, et al. Catching errors with in vivoEPID dosimetry. Med Phys. 2010;37(6):2638-2644.

20. Olaciregui-Ruiz I, Rozendaal R, Mijnheer B, van Herk M, Mans A. Automatic in vivo portal dosimetry of all treatments. Phys Med Biol. 2013;58(22):8253-64.

21. Rozendaal RA, Mijnheer BJ, van Herk M, Mans A. In vivo portal dosimetry for head-and-neck VMAT and lung IMRT: linking $\gamma$ analysis with differences in dose-volume histograms of the PTV. Radiother Oncol. 2014;112(3):396-401.

22. Celi S, Costa E, Wessels C, Mazal A, Fourquet A, Francois P. EPID based in vivo dosimetry system: clinical experience and results. J Appl Clin Med Phys. 2016;17(3):262-276. 
23. Podesta M, Persoon LC, Verhaegen F. A novel time dependent gamma evaluation function for dynamic $2 \mathrm{D}$ and $3 \mathrm{D}$ dose distributions. Phys Med Biol. 2014;59(20):5973-598.

24. Persoon LCGG, Nijsten SMJJG, Wilbrink FJ, et al. Interfractional trend analysis of dose differences based on 2D transit portal dosimetry. Phys Med Biol. 2012;57(20): 6445-6458.

25. Peerson LC, Egelmeer A, Öllers M, Nijsten S, Troost E, Verhaegen F. First clinical results of adaptive radiotherapy based on $3 \mathrm{D}$ portal dosimetry for lung cancer patients with atelectasis treated with volumetric-modulated arc therapy (VMAT). Acta Oncol. 2013;52(7):1484-1489.

26. Rozendaal RA, Mijnheer BJ, Hamming-Vrieze O, Mans A, van Herk M. Impact of daily anatomical changes on EPID-based in vivo dosimetry of VMAT treatments of head-and-neck cancer. Radiother Oncol. 2015;116(1):70-74.

27. Piermattei A, Greco F, Azario L, et al. A national project for in vivo dosimetry procedures in radiotherapy: first results. $\mathrm{Nucl}$ Instrum Methods Phys Res B. 2012;274:42-50.

28. Greco F, Piermattei A, Azario L, et al. aSi-EPID transit signal calibration for dynamic beams: a step for the IMRT in vivo dosimetry. Med Biol Eng Comput. 2013;51(10): 1137-1145.

29. Cilla S, Azario L, Greco F, et al. An in-vivo dosimetry procedure for Elekta step and shoot IMRT. Phys Med. 2014;10(4): 419-26.

30. Fidanzio A, Porcelli A, Azario L, et al. Quasi real time in vivo dosimetry for VMAT. Med Phys. 2014;41(6):062103.

31. Fidanzio A, Azario L, Greco F, Cilla S, Piermattei A. Routine EPID in-vivo dosimetry in a reference point for conformal radiotherapy treatments. Phys Med Biol. 2015;60(8):N141. doi:10.1088/00319155/60/8/N141.

32. Russo M, Piermattei A, Greco F, et al. Step-and-Shoot IMRT by Siemens Beams: An EPID dosimetry verification during treatment. Technol Cancer Res Treat. 2016;15(4):535-545.

33. Cilla S, Meluccio D, Fidanzio A, et al. Initial clinical experience with Epid-based in-vivo dosimetry for VMAT treatments of headand-neck tumors. Phys Med. 2016;32(1):52-58.

34. Piermattei A, Cilla S, Grimaldi L, et al. Integration between in vivo dosimetry and image guided radiotherapy for lung tumors. Med Phys. 2009;36(6):2206-2214.

35. Piermattei A, Fidanzio A, Cilla S, et al. Dose-guide radiotherapy for lung tumors. Med Biol Eng Comput. 2010;48(1):79-86.

36. International Atomic Energy Agency (2001) Absolute dose in external beam radiotherapy: an international code of practice for dosimetry based on standards of absorbed dose to water. IAEA TRS 398. 40 Best Medical Italy srl, Manuale SOFTDISO.

37. Best Medical Italy srl, Operative Manual SOFTDISO.

38. Low DA, Harms WB, Mutic S, Purdy JA. A technique for the quantitative evaluation of dose distributions. Med Phys. 1998; 25(5):656-661.

39. Joanes DN, Gill CA. Comparing measures of sample skewness and kurtosis. Journal of the Royal Statistical Society (Series D): The Statistician. 1998;47(1):183-189.

40. Dunlop A, McQuaid D, Nill S, et al. Comparison of CT number calibration techniques for CBCT-based dose calculation. Strahlenther Onkol. 2015;191(12):970-978.

41. Fidanzio A, Menna S, Greco F, et al. Dosimetric evaluation of new method for patient specific CBCT scan calibration. Radiother Oncol. 2016;119(1):S852. 